TOPOLOGICAL ALGEBRAS, THEIR APPLICATIONS,

AND RELATED TOPICS

BANACH CENTER PUBLICATIONS, VOLUME 67

INSTITUTE OF MATHEMATICS

POLISH ACADEMY OF SCIENCES

WARSZAWA 2005

\title{
ON MULTI-DIMENSIONAL GENERALIZATIONS OF THE WIENER-ŻELAZKO AND LÉVY-ŻELAZKO THEOREMS
}

\author{
S. ROLEWICZ \\ Institute of Mathematics, Polish Academy of Sciences \\ Śniadeckich 8, P.O. Box 21, 00-956 Warszawa 10, Poland \\ E-mail: rolewicz@impan.gov.pl
}

Abstract. Multi-dimensional generalizations of the Wiener-Żelazko and Lévy-Żelazko theorems are obtained.

We shall start with the classical Wiener theorem.

Wiener TheOrem (Wiener (1933)). Let $f(t)=\sum_{n \in \mathbb{Z}} a_{n} e^{\text {int }}$, where $\mathbb{Z}$ is the set of all integers, be a periodic function. Suppose that

$$
\|f\|_{1}=\sum_{n \in \mathbb{Z}}\left|a_{n}\right|<+\infty
$$

If $f(t) \neq 0$ for all $t$, then the function $1 / f$ can be developed in a trigonometric series $1 / f(t)=\sum_{n \in \mathbb{Z}} b_{n} e^{i n t}$ such that

$$
\left\|\frac{1}{f}\right\|_{1}=\sum_{n \in \mathbb{Z}}\left|b_{n}\right|<+\infty .
$$

A little later the following generalization of the Wiener theorem was given by Lévy. LÉvy THEOREM (Lévy (1933), (1934)). Let $f(t)=\sum_{n \in \mathbb{Z}} a_{n} e^{i n t}$ be a periodic function. Suppose that (1) holds. Let $\Phi(z)$ be an analytic function defined on an open set $U$ containing the image of $f$. Then the function $\Phi(f)$ can be developed in a trigonometric series $\Phi(f(t))=\sum_{n \in \mathbb{Z}} c_{n} e^{i n t}$ such that

$$
\|\Phi(f)\|_{1}=\sum_{n \in \mathbb{Z}}\left|c_{n}\right|<+\infty .
$$

The method of proof of the above mentioned theorems was simple classical analysis calculations.

2000 Mathematics Subject Classification: Primary 46H99; Secondary 42B05, 30B50, 46H20.

Key words and phrases: Fourier coefficients, coefficients of analytic functions, tensor products of $p$-normed algebras.

The paper is in final form and no version of it will be published elsewhere. 
The new method of proofs of the theorems of Wiener and Lévy was obtained via Banach algebras. The theory of Banach algebras started from the paper of Mazur (1938) and the famous paper of Gelfand (1941).

Żelazko (1960), (1962), (1965) extended this theory to locally bounded algebras. We recall that in locally bounded spaces the topology can be introduced by an equivalent $p$-homogeneous norm $\|\cdot\|$, i.e. such that

(1) $\|x\|=0$ if and only if $x=0$,

(2) $\|x+y\| \leq\|x\|+\|y\|$,

(3) there is $p, 0<p \leq 1$ such that $\|t x\|=|t|^{p}\|x\|$

(Aoki (1942), Rolewicz (1957)).

Żelazko introduced a new norm $\|\cdot\|_{Z}$ by the formula

$$
\|x\|_{Z}=\sup _{y \neq 0} \frac{\|x y\|}{\|y\|} .
$$

It is easy to see that $\|\cdot\|_{Z}$ is a $p$-homogeneous submultiplicative norm equivalent to the norm $\|\cdot\|($ Żelazko (1960)). Algebras with $p$-homogeneous norms are called $p$-normed algebras. The foundations of the theory of $p$-normed algebras were laid by Żelazko in his papers (1960), (1962), (1965).

As an immediate consequence we obtain the Wiener-Żelazko theorem.

Wiener-ŻELAZKo Theorem (Żelazko (1960)). Let $f(t)=\sum_{n \in \mathbb{Z}} a_{n} e^{\text {int }}$ be a periodic function. Suppose that there is $\mathbf{p}, 0<\mathbf{p} \leq 1$, such that

$$
\|f\|_{\mathbf{p}}=\sum_{n \in \mathbb{Z}}\left|a_{n}\right|^{\mathbf{p}}<+\infty .
$$

If $f(t) \neq 0$ for all $t$, then the function $1 / f$ can be developed in a trigonometric series $1 / f(t)=\sum_{n \in \mathbb{Z}} b_{n} e^{i n t}$ such that

$$
\left\|\frac{1}{f}\right\|_{\mathbf{p}}=\sum_{n \in \mathbb{Z}}\left|b_{n}\right|^{\mathbf{p}}<+\infty .
$$

More complicated was the extension of the proof of the Lévy theorem. The reason was that if a space $X$ is not locally convex, then the Riemann integrals of the function with values in $X$ may not exist. Even more, if such an integral exists for an arbitrary continuous function, then the space $X$ is locally convex (Mazur and Orlicz (1948)). Nevertheless, using some per pedes method Żelazko proved

LÉVY-ŻELAZKo ThEOREM (Żelazko (1962)). Let $f(t)=\sum_{n \in \mathbb{Z}} a_{n} e^{i n t}$ be a periodic function. Suppose that $(1)_{\mathbf{p}}$ holds. Let $\Phi(z)$ be an analytic function defined on an open set $U$ containing the image of $f$. Then the function $\Phi(f)$ can be developed in a trigonometric series $\Phi(f(t))=\sum_{n \in \mathbb{Z}} c_{n} e^{i n t}$ such that

$$
\|\Phi(f)\|_{\mathbf{p}}=\sum_{n \in \mathbb{Z}}\left|c_{n}\right|^{\mathbf{p}}<+\infty
$$

Till now we have considered only $n$ being an integer. The Wiener-Żelazko and LévyŻelazko theorems can be extended to the case of functions of $k$ variables. For this purpose we need a notion of tensor product of $p$-normed algebras. Let us recall the basic definitions. 
Let $\left(X^{j},\|\cdot\|_{j}\right), j=1, \ldots, k$, be $p$-normed spaces. By the tensor product $X^{1} \otimes \ldots \otimes X^{k}$ of those spaces we mean the set of formal sums of elements $x_{1} \otimes \ldots \otimes x_{k}$, i.e. elements of the form

$$
u=\sum_{i=1}^{n} x_{1}^{u, i} \otimes \ldots \otimes x_{k}^{u, i},
$$

where $x_{j}^{u, i} \in X^{j}, j=1, \ldots, k$.

On the tensor product $X^{1} \otimes \ldots \otimes X^{k}$ we can introduce a $p$-homogeneous norm $\|\cdot\|_{\otimes}$ in the following way:

$$
\|u\|_{\otimes}=\inf \sum_{i=1}^{n}\left\|x_{1}^{u, i}\right\|_{1} \ldots\left\|x_{k}^{u, i}\right\|_{k},
$$

where the infimum is taken over all representations $u=\sum_{i=1}^{n} x_{1}^{u, i} \otimes \ldots \otimes x_{k}^{u, i}$ (see Mallios (1986), for normed spaces Schatten (1950), Köthe (1979)).

It is not difficult to check that the above norm is also $p$-homogeneous. Thus $X^{1} \otimes \ldots \otimes$ $X^{k}$ is a $p$-normed space. This space need not be complete. We shall denote its completion by $X^{1} \hat{\otimes} \ldots \hat{\otimes} X^{k}$.

Suppose now that $\left(X^{j},\|\cdot\|_{j}\right), j=1, \ldots, k$, are $p$-normed algebras. Then we can introduce multiplication in the following way:

$$
\begin{aligned}
u * w & =\left(\sum_{i=1}^{n} x_{1}^{u, i} \otimes \ldots \otimes x_{k}^{u, i}\right) *\left(\sum_{j=1}^{m} x_{1}^{w, j} \otimes \ldots \otimes x_{k}^{w, j}\right) \\
& :=\sum_{i=1}^{n} \sum_{j=1}^{m} x_{1}^{u, i} x_{1}^{w, j} \otimes \ldots \otimes x_{k}^{u, i} x_{k}^{w, j} .
\end{aligned}
$$

It is not difficult to observe that with this multiplication $X^{1} \otimes \ldots \otimes X^{k}$ and $X^{1} \hat{\otimes} \ldots \hat{\otimes} X^{k}$ are $p$-normed algebras. If all the algebras $X^{j}, j=1, \ldots, k$, are commutative, then the algebras $X^{1} \otimes \ldots \otimes X^{k}$ and $X^{1} \hat{\otimes} \ldots \hat{\otimes} X^{k}$ are commutative, too.

Let $0<\mathbf{p} \leq 1$ and let

$$
\ell^{\mathbf{p}}=\left\{x=\left\{x_{n}\right\}: n=0, \pm 1, \pm 2, \ldots\right\}
$$

with p-homogeneous norm

$$
\|x\|_{\mathbf{p}}=\sum_{n \in \mathbb{Z}}\left|x_{n}\right|^{\mathbf{p}}
$$

It is not difficult to show that $\ell^{\mathbf{p}}$ is a complete locally bounded space.

In $\ell^{\mathbf{p}}$ we can introduce convolution multiplication

$$
a * b=\left\{\sum_{i \in \mathbb{Z}} a_{i} b_{n-i}\right\}
$$

and the norm $\|\cdot\|_{\mathbf{p}}$ is submultiplicative.

The algebra $\ell^{\mathbf{p}}$ can be considered also as an algebra $\mathcal{F}_{\mathbf{p}}$ of periodic (with period $2 \pi$ ) functions $f^{a}(t)=\sum_{n \in \mathbb{Z}} a_{n} e^{i n t}$. In this case multiplication is just the classical pointwise multiplication, $f^{a * b}(t)=f^{a}(t) f^{b}(t)$.

It can be shown that all multiplicative linear functionals $\mathbf{F}(\cdot)$ on the algebra $\ell^{\mathbf{p}}$ are of the form $\mathbf{F}(a)=f^{a}\left(t_{0}\right)$, where $0 \leq t_{0}<2 \pi$ (Żelazko (1960)). 
Now we shall consider the tensor product of $k$ algebras $\ell^{\mathbf{p}}, \mathcal{A}_{\mathbf{p}, k}=\ell^{\mathbf{p}} \otimes \ldots \otimes \ell^{\mathbf{p}}$. It is the algebra of all sequences $a=\left\{a_{\mathbf{n}}\right\}=\left\{a_{n_{1}, n_{2}, \ldots, n_{k}}\right\}$, where $n_{i}=0, \pm 1, \pm 2, \ldots$ for $i=1,2, \ldots, k$, with convolution multiplication

$$
a * b=\left\{\sum_{\mathbf{i} \in \mathbb{Z}^{k}} a_{\mathbf{i}} b_{\mathbf{n}-\mathbf{i}}\right\} .
$$

It is easy to observe that the algebras $\mathcal{A}_{\mathbf{p}, k}$ can also be interpreted as algebras $\mathcal{F}_{\mathbf{p}, k}$ of continuous functions of $k$ variables defined on $\mathbb{R}^{k}$, which are periodic with period $2 \pi$ with respect to each variable.

Observe that the convolution multiplication in the algebra $\mathcal{A}_{\mathbf{p}, k}$ gives pointwise multiplication of functions in the algebra $\mathcal{F}_{\mathbf{p}, k}$,

$$
f^{a * b}(\mathbf{t})=f^{a}(\mathbf{t}) f^{b}(\mathbf{t}) .
$$

From the general form of multiplicative linear functionals in the algebra $\ell^{\mathbf{p}}$ and the representation of this algebra as an algebra of functions with pointwise multiplication we immediately obtain

THEOREM 1. All multiplicative linear functionals $\mathbf{F}(\cdot)$ on the algebra $\mathcal{A}_{\mathbf{p}, k}$ are of the form $\mathbf{F}(a)=f^{a}\left(\mathbf{t}^{0}\right)=f^{a}\left(t_{1}^{0}, \ldots, t_{k}^{0}\right)$, where $0 \leq t_{i}^{0}<2 \pi, i=1, \ldots, k$.

Thus by the Żelazko theorem ('̇elazko 1960) we get that the spectrum of an element $a$ belonging to the algebra $\mathcal{A}_{\mathbf{p}, k}$ is nothing other than the set of values of the corresponding function $f^{a} \in \mathcal{F}_{\mathbf{p}, k}$. As an obvious consequence we get the following multi-dimensional generalization of the Wiener-Żelazko theorem.

COROllary 2. Let $f(\mathbf{t})=f\left(t_{1}, \ldots, t_{k}\right)$ be a $2 \pi$-periodic function with respect to each variable, represented by a series

$$
f(\mathbf{t})=\sum_{\mathbf{n} \in \mathbb{Z}^{k}} a_{\mathbf{n}} e^{i(\mathbf{n}, \mathbf{t})} .
$$

Suppose that for a certain $\mathbf{p}, 0<\mathbf{p} \leq 1$,

$$
\sum_{\mathbf{n} \in \mathbb{Z}^{k}}\left|a_{\mathbf{n}}\right|^{\mathbf{p}}<+\infty
$$

If $f(\mathbf{t}) \neq 0$ on the whole set $\mathbb{R}^{k}$ then $1 / f$ can be represented as a series

$$
\frac{1}{f(\mathbf{t})}=\sum_{\mathbf{n} \in \mathbb{Z}^{k}} b_{\mathbf{n}} e^{i(\mathbf{n}, \mathbf{t})}, \quad \text { where } \sum_{\mathbf{n} \in \mathbb{Z}^{k}}\left|b_{\mathbf{n}}\right|^{\mathbf{p}}<+\infty .
$$

The following corollary is the multi-dimensional generalization of the Lévy-Żelazko theorem.

Corollary 3. Let $f(\mathbf{t})=f\left(t_{1}, \ldots, t_{k}\right)$ be a $2 \pi$-periodic function with respect to each variable, represented by a series

$$
f(\mathbf{t})=\sum_{\mathbf{n} \in \mathbb{Z}^{k}} a_{\mathbf{n}} e^{i(\mathbf{n}, \mathbf{t})} .
$$

Suppose that for a certain $\mathbf{p}, 0<\mathbf{p} \leq 1$,

$$
\sum_{\mathbf{n} \in \mathbb{Z}^{k}}\left|a_{\mathbf{n}}\right|^{\mathbf{p}}<+\infty
$$


Let $\Phi(z)$ be an analytic function defined on an open set $U$ containing the image of the function $f$. Then $\Phi(f)$ is a periodic function with respect to each variable, defined on $\mathbb{R}^{k}$ of the form

$$
\Phi(f(\mathbf{t}))=\sum_{\mathbf{n} \in \mathbb{Z}^{k}} c_{\mathbf{n}} e^{i(\mathbf{n}, \mathbf{t})}, \quad \text { where } \sum_{\mathbf{n} \in \mathbb{Z}^{k}}\left|c_{\mathbf{n}}\right|^{\mathbf{p}}<+\infty .
$$

There are several possibilities of generalizing Theorem 1 and Corollaries 2 and 3 . The first one is to replace the function $t^{p}$ by some other function.

Let $N(u):[0,+\infty) \rightarrow[0,+\infty)$ be a non-decreasing function such that $N(u)=0$ only for $u=0$. Suppose that for sufficiently small $u, v$,

(a) $N(u+v) \leq N(u)+N(v)$

(b) there is $C>0$ such that $N(u v) \leq C N(u) N(v)$,

(c) there are a real number $p, 0<p \leq 1$, and a convex function $N_{0}(\cdot)$ such that $N(u)=$ $N_{0}\left(u^{p}\right)$.

In further considerations we shall assume that the function $N(\cdot)$ has properties (a), (b), (c) mentioned above.

By $N(\ell)$ (Rolewicz (1985)) we shall denote the algebra of all sequences $\left\{a=\left\{a_{n}\right\}\right.$ : $n=0, \pm 1, \pm 2, \ldots\}$ with convolution multiplication

$$
a * b=\left\{\sum_{i \in \mathbb{Z}} a_{i} b_{n-i}\right\} .
$$

Now we shall consider the tensor product of $k$ algebras $N(\ell), \mathcal{A}_{N, k}=N(\ell) \otimes \ldots \otimes N(\ell)$. It is the algebra of all sequences $a=\left\{a_{\mathbf{n}}\right\}$, where $\mathbf{n}=\left(n_{1}, n_{2}, \ldots, n_{k}\right), n_{i}=0, \pm 1, \pm 2, \ldots$ for $i=1,2, \ldots, k$, with convolution multiplication

$$
a * b=\left\{\sum_{\mathbf{i} \in \mathbb{Z}^{k}} a_{\mathbf{i}} b_{\mathbf{n}-\mathbf{i}}\right\} .
$$

The algebra $N(\ell)$ can also be considered as the algebra $\mathcal{F}_{N, k}$ of analytic functions $2 \pi$-periodic in each variable.

Observe that the convolution multiplication in $\mathcal{A}_{N, k}$ gives pointwise multiplication of functions in $\mathcal{F}_{N, k}$,

$$
f^{a * b}(\mathbf{t})=f^{a}(\mathbf{t}) f^{b}(\mathbf{t}) .
$$

From the general form of multiplicative linear functionals in the algebra $N(\ell)$ and the representation of this algebra as an algebra of functions with pointwise multiplication we immediately obtain

ThEOREM 4. All multiplicative linear functionals $\mathbf{F}(\cdot)$ on the algebra $\mathcal{A}_{N, k}$ are of the form $\mathbf{F}(a)=f^{a}\left(\mathbf{t}^{0}\right)=f^{a}\left(t_{1}^{0}, \ldots, t_{k}^{0}\right)$, where $0 \leq t_{i}^{0}<2 \pi, i=1, \ldots, k$.

Thus by the Żelazko theorem (Żelazko 1960) we get that the spectrum of an element $a$ belonging to the algebra $\mathcal{A}_{N, k}$ is just the set of values of the corresponding function $f^{a} \in$ $\mathcal{F}_{N, k}$. As an obvious consequence we get the following multi-dimensional generalization of the Wiener-Żelazko theorem. 
COROllary 5. Let $f(\mathbf{t})=f\left(t_{1}, \ldots, t_{k}\right)$ be $2 \pi$-periodic with respect to each variable and represented by a series

$$
f(\mathbf{t})=\sum_{\mathbf{n} \in \mathbb{Z}^{k}} a_{\mathbf{n}} e^{i(\mathbf{n t})}, \text { where } \sum_{\mathbf{n} \in \mathbb{Z}^{k}} N\left(\left|a_{\mathbf{n}}\right|\right)<+\infty .
$$

If $f(\mathbf{t}) \neq 0$ on the whole set $\mathbb{R}^{k}$ then $1 / f$ can be represented by a series

$$
\frac{1}{f(\mathbf{t})}=\sum_{\mathbf{n} \in \mathbb{Z}^{k}} b_{\mathbf{n}} e^{i(\mathbf{n}, \mathbf{t})}, \quad \text { where } \sum_{\mathbf{n} \in \mathbb{Z}^{k}} N\left(\mid\left(b_{\mathbf{n}} \mid\right)<+\infty .\right.
$$

The following corollary is the multi-dimensional generalization of the Lévy-Żelazko theorem.

COROllary 6. Let $f(\mathbf{t})=f\left(t_{1}, \ldots, t_{k}\right)$ be $2 \pi$-periodic with respect to each variable and represented by a series

$$
f(\mathbf{t})=\sum_{\mathbf{n} \in \mathbb{Z}^{k}} a_{\mathbf{n}} e^{i(\mathbf{n}, \mathbf{t})}, \quad \text { where } \sum_{\mathbf{n} \in \mathbb{Z}^{k}} N\left(\left|a_{\mathbf{n}}\right|\right)<+\infty .
$$

Let $\Phi$ be an analytic function defined on an open set $U$ containing the image of the function $f$. Then $\Phi(f)$ is a periodic function with respect to each variable defined on $\mathbb{R}^{k}$ of the form

$$
\Phi(f(\mathbf{t}))=\sum_{\mathbf{n} \in \mathbb{Z}^{k}} c_{\mathbf{n}} e^{i(\mathbf{n}, \mathbf{t})}, \quad \text { where } \sum_{\mathbf{n} \in \mathbb{Z}^{k}} N\left(\left|c_{\mathbf{n}}\right|\right)<+\infty .
$$

The results given above can be extended to the case of analytic functions.

By $N^{+}(\ell)$ we shall denote the algebra of all sequences $\left\{a=\left\{a_{m}\right\}: m=0,1,2, \ldots\right\}$ with convolution multiplication

$$
a * b=\left\{\sum_{i \in \mathbb{N}} a_{i} b_{m-i}\right\},
$$

where $\mathbb{N}$ denotes the set of non-negative integers.

With this multiplication $N^{+}(\ell)$ is a commutative complete $p$-normed algebra (see Rolewicz (1985)).

The algebra $N^{+}(\ell)$ can be considered also as the algebra $\mathcal{F}_{N^{+}}$of analytic functions $f^{a}(z)=\sum_{m \in \mathbb{N}} a_{m} z^{m}$ defined on the unit disc $\mathbb{D}=\{z:|z| \leq 1\}$. In this case, as in the previous cases, multiplication is just the classical pointwise multiplication, $f^{a * b}(z)=$ $f^{a}(z) f^{b}(z)$.

It can be shown that all multiplicative linear functionals $\mathbf{F}(\cdot)$ defined on the algebra $N^{+}(\ell)$ are of the form $\mathbf{F}(a)=f^{a}\left(z_{0}\right)$, where $\left|z_{0}\right| \leq 1$ (see Rolewicz (1985)).

Now we shall consider the tensor product $\mathcal{A}_{N, k^{\prime}}^{\prime}=N^{+}(\ell) \otimes \ldots \otimes N^{+}(\ell)$ of $k^{\prime}$ algebras $N^{+}(\ell)$. It is an algebra of all sequences $a=\left\{a_{\mathbf{m}}\right\}=\left\{a_{m_{1}, n_{2}, \ldots, m_{k}}\right\}$, where $m_{i}=0,1,2, \ldots$ for $i=1,2, \ldots, k^{\prime}$, with convolution multiplication

$$
a * b=\left\{\sum_{i \in \mathbb{N}} a_{\mathbf{i}} b_{\mathbf{m}-\mathbf{i}}\right\} .
$$

It is easy to observe that the algebras $\mathcal{A}_{N, k^{\prime}}^{\prime}$ can be also interpreted as algebras $\mathcal{F}_{N, k^{\prime}}^{\prime}$ of analytic functions defined on $\mathbb{D}^{k^{\prime}}$. 
From the general form of multiplicative linear functionals on the algebra $N^{+}(\ell)$ and the representation of this algebra as an algebra of functions with pointwise multiplication we immediately obtain

THEOREM 7. All multiplicative linear functionals $\mathbf{F}(\cdot)$ on the algebra $\mathcal{A}_{N, k^{\prime}}^{\prime}$ are of the form $\mathbf{F}(a)=f^{a}\left(\mathbf{z}^{0}\right)$, where $\mathbf{z}^{0}=\left(z_{1}^{0}, \ldots, z_{k^{\prime}}^{0}\right) \in \mathbb{D}^{k^{\prime}}$.

As an obvious consequence we get

Corollary 8. Let $f(\mathbf{z})$ be an analytic function defined on $\mathbb{D}^{k^{\prime}}$ and represented by a series

$$
f(\mathbf{z})=\sum_{m \in \mathbb{N}^{\prime}} a_{\mathbf{m}} \mathbf{z}^{\mathbf{m}}
$$

where we denote $\mathbf{z}^{\mathbf{m}}=: z_{1}^{m_{1}} \ldots z_{k^{\prime}}^{m_{k^{\prime}}}$. Suppose that

$$
\sum_{m \in \mathbb{N}^{k^{\prime}}} N\left(\left|a_{\mathbf{m}}\right|\right)<+\infty .
$$

If $f(\mathbf{z}) \neq 0$ on the whole set $\mathbb{D}^{k^{\prime}}$, then $1 / f$ can be represented by a series

$$
\frac{1}{f(\mathbf{z})}=\sum_{m \in \mathbb{N} k^{\prime}} b_{\mathbf{m}} \mathbf{z}^{\mathbf{m}}, \quad \text { where } \sum_{m \in \mathbb{N}^{\prime}} N\left(\left|b_{\mathbf{m}}\right|\right)<+\infty .
$$

Corollary 9. Let $f(\mathbf{z})$ be an analytic function defined on $\mathbb{D}^{k^{\prime}}$ and represented by a series

$$
f(\mathbf{z})=\sum_{m \in \mathbb{N}^{k^{\prime}}} a_{\mathbf{m}} \mathbf{z}^{\mathbf{m}}, \quad \text { where } \sum_{m \in \mathbb{N}^{k^{\prime}}} N\left(\left|a_{\mathbf{m}}\right|\right)<+\infty .
$$

Let $\Phi(z)$ be an analytic function defined on an open set $U$ containing the image of the function $f$. Then the function $\Phi(f)$ is analytic on $\mathbb{D}^{k^{\prime}}$ and it can be represented by a series

$$
\Phi(f(\mathbf{z}))=\sum_{m \in \mathbb{N} k^{\prime}} c_{\mathbf{m}} \mathbf{z}^{\mathbf{m}}, \quad \text { where } \sum_{m \in \mathbb{N} k^{\prime}} N\left(\left|c_{\mathbf{m}}\right|\right)<+\infty .
$$

Taking the tensor product $\mathcal{A}_{N, k, k^{\prime}}=\mathcal{A}_{N, k} \hat{\otimes} \mathcal{A}_{N, k^{\prime}}^{\prime}$ and $\mathcal{A}_{N, k^{\prime}}^{\prime}$ and using the method described above we obtain

Proposition 10. Let $f(\mathbf{t}, \mathbf{z})$, where $\mathbf{t}=\left(t_{1}, \ldots, t_{k}\right)$ and $\mathbf{z}=\left(z_{1}, \ldots, z_{k^{\prime}}\right)$, be a $2 \pi$ periodic function with respect to each of $t_{1}, \ldots, t_{k}$ and analytic with respect to $z_{1}, \ldots, z_{k^{\prime}}$, defined on $\mathbb{R}^{k^{\prime}} \times \mathbb{D}^{k^{\prime}}$ and represented by a series

$$
f(\mathbf{t}, \mathbf{z})=\sum_{\mathbf{n} \in \mathbb{Z}^{k}, \mathbf{m} \in \mathbb{N}^{k^{\prime}}} a_{\mathbf{n}, \mathbf{m}} e^{i(\mathbf{n}, \mathbf{t})} \mathbf{z}^{\mathbf{m}}, \quad \text { where } \sum_{\mathbf{n} \in \mathbb{Z}^{k}, \mathbf{m} \in \mathbb{N}^{k^{\prime}}} N\left(\left|a_{\mathbf{n}, \mathbf{m}}\right|\right)<+\infty .
$$

If $f(\mathbf{t}, \mathbf{z}) \neq 0$ on the whole set $\mathbb{R}^{k^{\prime}} \times \mathbb{D}^{k^{\prime}}$, then $1 / f$ can be represented by a series

$$
\frac{1}{f(\mathbf{t}, \mathbf{z})}=\sum_{\mathbf{n} \in \mathbb{Z}^{k}, \mathbf{m} \in \mathbb{N}^{k^{\prime}}} b_{\mathbf{n}, \mathbf{m}} e^{i(\mathbf{n}, \mathbf{t})} \mathbf{z}^{\mathbf{m}}, \quad \text { where } \sum_{\mathbf{n} \in \mathbb{Z}^{k}, \mathbf{m} \in \mathbb{N}^{k^{\prime}}} N\left(\left|b_{\mathbf{n}, \mathbf{m}}\right|\right)<+\infty .
$$

Proposition 11. Let $f(\mathbf{t}, \mathbf{z})$, where $\mathbf{t}=\left(t_{1}, \ldots, t_{k}\right)$ and $\mathbf{z}=\left(z_{1}, \ldots, z_{k^{\prime}}\right)$, be a $2 \pi$ periodic function with respect to each of $t_{1}, \ldots, t_{k}$ and analytic with respect to $z_{1}, \ldots, z_{k^{\prime}}$, 
defined on $\mathbb{R}^{k^{\prime}} \times \mathbb{D}^{k^{\prime}}$ and represented by a series

$$
f(\mathbf{t}, \mathbf{z})=\sum_{\mathbf{n} \in \mathbb{Z}^{k}, \mathbf{m} \in \mathbb{N}^{k^{\prime}}} a_{\mathbf{n}, \mathbf{m}} e^{i(\mathbf{n}, \mathbf{t})} \mathbf{z}^{\mathbf{m}}, \text { where } \sum_{\mathbf{n} \in \mathbb{Z}^{k}, \mathbf{m} \in \mathbb{N}^{k^{\prime}}} N\left(\left|a_{\mathbf{n}, \mathbf{m}}\right|\right)<+\infty .
$$

Let $\Phi$ be an analytic function defined on an open set $U$ containing the image of the function $f$. Then $\Phi(f)$ is a function periodic with respect to $\mathbf{t}$ and analytic with respect to $\mathbf{z}$, defined on $\mathbb{R}^{k} \times \mathbb{D}^{k^{\prime}}$ of the form

$$
\Phi(f((\mathbf{t}, \mathbf{z})))=\sum_{\mathbf{n} \in \mathbb{Z}^{k}, \mathbf{m} \in \mathbb{N}^{k^{\prime}}} c_{\mathbf{n}, \mathbf{m}} e^{i(\mathbf{n}, \mathbf{t})} \mathbf{z}^{\mathbf{m}}, \text { where } \sum_{\mathbf{n} \in \mathbb{Z}^{k}, \mathbf{m} \in \mathbb{N}^{k^{\prime}}} N\left(\left|c_{\mathbf{n}, \mathbf{m}}\right|\right)<+\infty .
$$

Till now we have considered multidimensional generalizations of the Lévy-Żelazko theorem for the function $\Phi$ of one complex variable.

Using integration of analytic functions with values in $p$-normed spaces (see Gramsch (1965), (1967), Przeworska-Rolewicz and Rolewicz (1966)) we can generalize Proposition 11 to the case of analytic functions $\Phi\left(z_{1}, \ldots, z_{r}\right)$ of $r$ complex variables and get

Proposition 12. Let $f^{j}(\mathbf{t}, \mathbf{z}), j=1, \ldots, r$, where $\mathbf{t}=\left(t_{1}, \ldots, t_{k}\right)$ and $\mathbf{z}=\left(z_{1}, \ldots, z_{k^{\prime}}\right)$, be $2 \pi$-periodic functions with respect to each of $t_{1}, \ldots, t_{k}$ and analytic with respect to $z_{1}, \ldots, z_{k^{\prime}}$, defined on $\mathbb{R}^{k^{\prime}} \times \mathbb{D}^{k^{\prime}}$ and represented by a series

$$
f^{j}(\mathbf{t}, \mathbf{z})=\sum_{\mathbf{n} \in \mathbb{Z}^{k}, \mathbf{m} \in \mathbb{N}^{k^{\prime}}} a_{\mathbf{n}, \mathbf{m}}^{j} e^{i(\mathbf{n}, \mathbf{t})} \mathbf{z}^{\mathbf{m}} .
$$

Suppose that for each $j=1, \ldots, r$,

$$
\sum_{\mathbf{n} \in \mathbb{Z}^{k}, \mathbf{m} \in \mathbb{N}^{k^{\prime}}} N\left(\left|a_{\mathbf{n}, \mathbf{m}}^{j}\right|\right)<+\infty .
$$

Let $\Phi\left(z_{1}, \ldots, z_{r}\right)$ be an analytic function defined on an open set $U$ containing the image of the vector function $\mathbf{f}=\left(f_{1}, \ldots, f_{r}\right)$. Then $\Phi(\mathbf{f}(\mathbf{t}, \mathbf{z}))$ is a function periodic with respect to $\mathbf{t}$ and analytic with respect to $\mathbf{z}$, defined on $\mathbb{R}^{k} \times \mathbb{D}^{k^{\prime}}$ of the form

$$
\Phi(f((\mathbf{t}, \mathbf{z})))=\sum_{\mathbf{n} \in \mathbb{Z}^{k}, \mathbf{m} \in \mathbb{N}^{k^{\prime}}} c_{\mathbf{n}, \mathbf{m}} e^{i(\mathbf{n}, \mathbf{t})} \mathbf{z}^{\mathbf{m}}, \text { where } \sum_{\mathbf{n} \in \mathbb{Z}^{k}, \mathbf{m} \in \mathbb{N}^{k^{\prime}}} N\left(\left|c_{\mathbf{n}, \mathbf{m}}\right|\right)<+\infty .
$$

For $N(t)=t$ Proposition 12 can be easily deduced from the theorems of Shilov (1951) and Arens-Calderón (1955). For $k=1$ and $N(t)=t^{p}, 0<p<1$ it was proved by Przeworska-Rolewicz and Rolewicz (1966) and Gramsch (1967) (see also Rolewicz (1985)).

\section{References}

T. Aoki (1942), Locally bounded linear topological spaces, Proc. Imp. Acad. Tokyo 18, 588-594.

R. Arens and A. P. Calderón (1955), Analytic functions of several Banach algebra elements, Annals of Math. 62, 204-216.

I. M. Gelfand (1941), Normierte Ringe, Mat. Sb. 9, 3-24.

B. Gramsch (1965), Integration und holomorphe Funktionen in lokalbeschränkte Räumen, Math. Ann. 162, 190-210.

B. Gramsch (1967), Funktionalkalkül mehrerer Veränderlichen in lokalbeschränkte Algebren, Math. Ann. 174, 311-344. 
G. Köthe (1979), Topological Vector Spaces, Grundlehren Mathematischen Wissenschaften 237, Springer.

P. Lévy (1933), Sur la convergence des séries de Fourier, CR Paris 196, 463-464.

P. Lévy (1934), Sur la convergence des séries de Fourier, Compositio Math. 1, 1-14.

A. Mallios (1986), Topological Algebras. Selected Topics, North-Holland Math. Studies 124, North-Holland.

S. Mazur (1938), Sur les anneaux linéaires, C. R. Acad. Sci. Paris 207, 1025-1027.

D. Przeworska-Rolewicz and S. Rolewicz (1966), On integrals of functions with values in linear metric spaces, Stud. Math. 26, 121-131.

S. Rolewicz (1957), On a certain class of linear metric spaces, Bull. Acad. Pol. Sc. 5, 471-473.

S. Rolewicz (1985), Metric Linear Spaces, PWN-Polish Scientific Publishers and D. Reidel, Warszawa-Dordrecht, 2-nd revised and extended edition.

G. E. Shilov (1951), On the rings of functions with uniform convergence (in Russian), Ukrain. Math. Ž. 3 (4), 404-411.

R. Schatten (1950), A Theory of Cross-Spaces, Ann. of Math. Studies 26.

N. Wiener (1932), Tauberian theorems, Annals of Mathematics 33, 1-100.

W. Żelazko (1960), On the locally bounded and m-convex topological algebras, Stud. Math. 19, $333-356$.

W. Żelazko (1962), Analytic functions in p-normed algebras, Stud. Math. 21, 345-350.

W. Żelazko (1965), Metric generalizations of Banach algebras, Diss. Math. 47.

W. Żelazko (1973), Banach Algebras, Elsevier and PWN-Polish Scientific Publishers, Amsterdam-Warszawa.

A. Zygmund (1959), Trigonometric Series, 2nd ed., Cambridge Univ. Press. 